OPEN ACCESS

Edited by:

Jun Wang,

Icahn School of Medicine at Mount

Sinai, United States

Reviewed by:

Taru Goyal,

All India Institute of Medical Sciences

Jodhpur, India

Hsiao-Yun Lin,

Icahn School of Medicine at Mount

Sinai, United States

*Correspondence:

Xuezhu Zhang

xzzhang@tjutcm.edu.cn

tThese authors have contributed

equally to this work

Received: 26 July 2021 Accepted: 27 October 2021

Published: 29 November 2021

Citation:

Zhi H, Wang Y, Chang S, Pan P, Ling Z, Zhang Z, Ma Z, Wang $R$ and

Zhang X (2021) Acupuncture Can

Regulate the Distribution

of Lymphocyte Subsets

and the Levels of Inflammatory

Cytokines in Patients With Mild

to Moderate Vascular Dementia.

Front. Aging Neurosci. 13:747673.

doi: 10.3389/fnagi.2021.747673

\section{Acupuncture Can Regulate the Distribution of Lymphocyte Subsets and the Levels of Inflammatory Cytokines in Patients With Mild to Moderate Vascular Dementia}

\author{
Hui Zhi',2t, Yao Wang ${ }^{1,2+}$, Shichen Chang 1,2, Pan Pan 1,2,3, Zhenzhen Ling ${ }^{1,4}$, Zhen Zhang ${ }^{1,5}$, \\ Zhinan Ma1,2, Runmin Wang ${ }^{1,2}$ and Xuezhu Zhang ${ }^{1,2 *}$ \\ ' First Teaching Hospital of Tianjin University of Traditional Chinese Medicine, Tianjin, China, ${ }^{2}$ National Clinical Research \\ Center for Chinese Medicine Acupuncture and Moxibustion, Tianjin, China, ${ }^{3}$ Second Clinical Medical College, Yunnan \\ University of Traditional Chinese Medicine, Kunming, China, ${ }^{4}$ Department of Immune Regulation, Immunology Frontier \\ Research Center, Osaka University, Osaka, Japan, ${ }^{5}$ Weifang Traditional Chinese Hospital, Weifang, China
}

Background: Vascular dementia (VD) is the second most common type of dementia after Alzheimer's disease, but there is a lack of definitive treatment for VD. Acupuncture treatment is effective in improving the cognitive impairment and behavioral capacity of patients with VD. In recent years, more studies indicated that peripheral inflammation and abnormal peripheral immune function may aggravate neuroinflammation and cognitive dysfunction. However, there are few studies about the acupuncture and the abnormal peripheral immune function of VD. Also, few studies concern the regulating effect of acupuncture on peripheral immunity of patients with VD.

Objective: The aim of this study was to explore the effect of the "sanjiao" acupuncture method on peripheral immunity of patients with mild to moderate VD.

Methods: A total of 30 patients with VD were involved in the acupuncture group (AG), which was treated with the "sanjiao" acupuncture method once a day for six times a week and lasted for 12 weeks, and 30 healthy elderly people were assigned to the normal group (NG), which had no treatment. The distribution of lymphocyte subsets and the levels of some inflammatory cytokines in the peripheral blood of subjects were evaluated using the flow cytometry (FCM) and the enzyme-linked immunosorbent assay (ELISA).

Results: A total of 60 subjects were involved in this study, while 58 subjects completed the entire trial. Before treatment, the levels of $\mathrm{CD}^{+} \mathrm{T}, \mathrm{CD} 4^{+} \mathrm{T}$ cells, $\mathrm{CD} 4^{+} / \mathrm{CD} 8^{+}$, Tregs, B cells, IFN- $\gamma$, and IL-10 in patients with VD were significantly decreased compared with the normal group (all $P<0.05$ or $P<0.01$ ). The level of TNF- $\alpha$ in peripheral blood of patients with VD was significantly increased $(P<0.01)$. After acupuncture treatment, the levels of $\mathrm{CD}^{+}{ }^{+} \mathrm{T}, \mathrm{CD} 4^{+} \mathrm{T}$ cells, and IFN- $\gamma$ were significantly increased (all $P<0.05$ or $P<0.01)$. The level of TNF- $\alpha$ was significantly decreased $(P<0.01)$. The proportion 
of Tregs was increased $(P<0.01)$, but it was still lower than that of the normal group $(P<0.05)$.

Conclusion: The acupuncture method can increase the proportion of $\mathrm{CD}^{+}, \mathrm{CD}^{+}$ $\mathrm{T}$ cells, and Tregs in peripheral blood of patients with VD. And, it reduces the levels of pro-inflammatory factor TNF- $\alpha$, which achieves the anti-inflammatory effects and immunostimulation. It suggests that acupuncture can improve the peripheral immune dysfunction of patients with VD by regulating the distribution of lymphocyte subsets and the levels of inflammatory cytokines.

Clinical Trial Registration: [www.chictr.org.cn], identifier [ChiCTR-IOR-17012052].

Keywords: vascular dementia, "sanjiao" acupuncture method, peripheral immune function, FCM, ELISA

\section{INTRODUCTION}

Vascular dementia (VD) is the general term of dementia syndrome caused by the injury of brain tissue resulting from a series of cerebral vascular diseases. Its clinical features are mainly cognitive dysfunction and neurological dysfunction, which are related to the cerebrovascular diseases. VD is the second most common type of dementia after Alzheimer's disease (AD), accounting for about $20 \%$ of all dementia populations (O'Brien and Thomas, 2015; Dichgans and Leys, 2017).

During the progress of $\mathrm{VD}$, chronic cerebral hypoperfusion $(\mathrm{CCH})$ and thromboembolism lead to a decrease in cerebral blood flow, causing anoxia, oxidative stress, and inflammatory response and finally leading to a cognitive defect (Venkat et al., 2015). Neuroinflammation plays a key role in the occurrence and progress of VD (Iadecola, 2010). After cerebral ischemia, the microglial cells are activated and release the proinflammatory factors, chemokines, and reactive oxygen species, which can damage the neuron. Meanwhile, these inflammatory mediators further promote the activation of microglial cells, which aggravate the inflammatory reaction. Otherwise, brain tissue releases high mobility group protein B1 (HMGB1), hypoxia-inducible factor-1 $\alpha$ (HIF-1 $\alpha$ ), brain-derived antigen, and other signals after ischemia which activate the peripheral immune system through the purinoceptors, Toll-like receptors, and receptors for activated glycation end products. Then, the lymphocyte subsets transform into the pro-inflammatory phenotype. Monocytes and macrophages are activated, and the pro-inflammatory factors are increased. All of these affect the immune function of patients with VD. Moreover, the immune cells such as neutrophils, monocyte, and macrophages in the peripheral blood and the pro-inflammatory factors that they released invade into brain parenchyma via damaged blood-brain barrier (BBB), further promoting the activation of microglial cells and aggravating the neuroinflammation which exacerbate the nerve injury.

Previously, the effect of inflammation of the central system on cognitive function is of great concern for a long time. However, in recent years, more studies indicate that peripheral inflammation and abnormal peripheral immune function may aggravate neuroinflammation and neuron damage (An et al., 2014; Prinz and Priller, 2017). More attention has been paid to the promotion of peripheral inflammation on the neuroinflammation, and the important role of the peripheral immune cells, especially $\mathrm{T}$ cells, in maintaining normal cognitive function.

For example, intraperitoneal injection of lipopolysaccharide (LPS), which induced peripheral inflammation, can cause behavioral changes and cognitive impairment in mice. Meanwhile, in the LPS-induced mice, the microglial cells are activated, neuronal cells are decreased, the NF-kB signaling pathway is activated, the expression of pro-inflammatory cytokines (TNF- $\alpha$ and IL-1 $\beta$ ) is increased, and the expression of anti-inflammatory cytokines (IL-4 and IL-10) is decreased (Zhao et al., 2019). The LPS cannot cross the blood-brain barrier (BBB), but the intraperitoneal injection of LPS can cause cognitive impairment. The peripheral inflammation may result in damages in the BBB, and peripheral immune cells infiltrate the brain parenchyma, causing neuroinflammation. The rats with hyperammonemia show a rapid and reversible induction of peripheral inflammation, with activation of astrocyte and microglial cells. Meanwhile, the expression of TNF- $\alpha$, IL-1 $\beta$, and gamma-aminobutyric acid (GABA) is increased, and the abilities of motor coordination and learning are impaired. These abnormal conditions can be prevented using peripheral anti-TNF- $\alpha$ treatment (Balzano et al., 2020).

$\mathrm{T}$ cells are the core of cellular immune response. When the body loses $\mathrm{T}$ cells, the $\mathrm{B}$ cells, mast cells, macrophages, and dendritic cells would transform into the pro-inflammatory phenotype, activate the immune system, release the proinflammatory factors into the brain and peripheral blood, and then inhibit the brain function. The cognitive function of rats with a shortage of $\mathrm{T}$ cells in peripheral blood is impaired, but the cognitive impairment can be improved after T-cell transplantation. However, the cognitive impairment induced by drugs and the abnormal behaviors can also have a recovery after T-cell transplantation (Radjavi et al., 2014). Research shows that the inhibition of $\mathrm{CD}^{+}{ }^{+} \mathrm{T}$ cells results in hippocampus neuron damage and cognitive impairment (De Miranda et al., 2017). These data indicate that peripheral inflammation and immunologic injury can cause and aggravate neuroinflammation, and result in cognitive impairment and many other changes. The $\mathrm{T}$ cells, especially $\mathrm{CD} 4^{+} \mathrm{T}$ cells, play a key role in maintaining the normal cognitive function. Otherwise, it is proved that the immune response plays an important role in cerebral small-vessel 
disease and VD (Raz et al., 2015). Many studies show that there are peripheral inflammation and abnormal peripheral immune function in VD, which include increased pro-inflammatory factors (IL-1 $\beta$, IL-6, TNF- $\alpha$, IFN- $\gamma$ ), decreased $T$ cells, the imbalance between the Th17 and Treg, and so on (Dadsetan et al., 2016; Balzano et al., 2019).

Professor Han Jingxian summed up his many years of clinical experience and created the "sanjiao" acupuncture method to treat VD and achieved good clinical results. Our previous studies had shown that the "sanjiao" acupuncture method could significantly improve the cognitive impairment and behavioral capacity of patients with VD (Yu et al., 2006). Some studies indicate that the acupuncture treatment has antiinflammatory and immunoregulation effects, and it is effective on VD (Shi et al., 2012; Chen et al., 2015, 2017; Si et al., 2016). Furthermore, acupuncture can improve the mitochondrial function of hippocampus and protect the nerves by the way of antioxidation, regulating cell proliferation, anti-apoptosis, etc. (Wang et al., 2009; Zhang et al., 2014).

At present, there are few studies about acupuncture and abnormal peripheral immune function of VD. And, few studies concern the regulating effect of acupuncture on the peripheral immunity of patients with VD. There is a close connection between the peripheral immune and the central nervous system, and regulating the peripheral immunity can influence the central nervous system and cognitive function. So, we think that regulating the peripheral immunity may be beneficial to the cognitive function of patients with VD. Therefore, this study investigates the regulatory effect of acupuncture treatment on peripheral immune function of patients with VD.

\section{MATERIALS AND METHODS}

\section{Trial Design}

This trial involved 30 patients with $\mathrm{VD}$ as the acupuncture group (AG), and 30 healthy elderly people were assigned to the normal group (NG). And, the trial lasted for 12 weeks. The distribution of lymphocyte subsets and the levels of some inflammatory cytokines in the peripheral blood of patients with VD before and after treatment were evaluated using FCM and ELISA. The distribution of lymphocyte subsets and the levels of some inflammatory cytokines in the peripheral blood of the healthy elderly people were also evaluated at the beginning of the trial. The study was performed according to the common guidelines for clinical trials (Declaration of Helsinki, International Conference on Harmonization/WHO Good Clinical Practice standards including certification by an external audit). The trial protocol was approved by the Research Ethical Committee of First Teaching Hospital of Tianjin University of Traditional Chinese Medicine. The research flow chart is shown in Figure 1.

\section{Participants}

This study included 30 patients with VD from the First Teaching Hospital of Tianjin University of Traditional Chinese Medicine. The inclusion criteria for VD referred to the National Institute for Neurological Disorders and Stroke (NINDS/AIREN) in 1993 and the Diagnostic and Statistical Manual of Mental Disorders (DSM-5). And, the determination of mild to moderate dementia was accorded to the Clinical Dementia Rating Table (CDR) criteria. Patients were also required to have a Mini-Mental State Examination (MMSE) score $\leq 24$ (above secondary school); $\leq 20$ (primary school level); $\leq 17$ (illiteracy), Hachinski Ischemic Scale score $\geq 7$, and Hamilton Depression Scale score $\leq 17$. Otherwise, patients who enrolled in this trial should be aged 55-80 years, and the female should be post-menopausal women with no fertility (at least 1 year after menopause). The principal exclusion criteria included the presence of other types of dementia or nerve and cerebral diseases (brain tumor, Parkinson's disease, multiple sclerosis, amyotrophy, myasthenia gravis, depressive syndrome, and severe neurological deficits). Patients suffering from severe heart, lung, liver, kidney, hematopoietic system, endocrine system diseases, and other diseases that can affect cognitive function were also excluded.

A total of 30 healthy elderly people whose gender and age matching with the AG were involved from the Physical Examination Center of the First Teaching Hospital of Tianjin University of Traditional Chinese Medicine. The exclusion criteria for healthy elderly people included cerebrovascular disease, dementia or other nervous system diseases, malignant tumor, severe hepatic, and renal dysfunction, hematopathy, HIV infection, recent bacterial or viral infection, chronic organic diseases, immunodeficiency disease, contact history of chemical poisons, and the personal history of current use of medications that can affect the immune system. Healthy elderly people who had hypertension, diabetes, coronary heart disease, and other internal medicine diseases that did not control stably and people who had psychogenia such as anxiety and depression were also excluded. All the participants signed the "informed consent form" and consented to undergo the relevant examination.

\section{Interventions}

In the $\mathrm{AG}$, the patients with VD received the "sanjiao" acupuncture method, which included Danzhong (CV17), Zhongwan (CV12), Qihai (CV6), bilateral Xuehai (SP10), Zusanli (ST36), and Waiguan (SJ5). One-off sterile acupuncture needles (Huatuo, Suzhou Medical Instruments Factory, Suzhou, China), with a length of $40 \mathrm{~mm}$ and diameter of $0.25 \mathrm{~mm}$, were used in the trial. Needles were inserted horizontally $15 \mathrm{~mm}$ into CV17 and SJ5; 25-40 mm perpendicularly into CV12, CV6, and ST36; 15-25 mm obliquely into SP10. After getting the needle sensation, the needles were retained in situ for $20 \mathrm{~min}$. The patients were given the acupuncture treatment once a day, 6 times a week for 12 weeks. And, the acupuncture therapy was performed by professional acupuncturists. The normal group had no interventions.

\section{Sample Collection and Evaluation}

At the beginning of the study, $4 \mathrm{ml}$ venous blood of every healthy individual in the normal group was collected in the EDTA tube for FCM and ELISA in the morning. Out of $4 \mathrm{ml}, 2 \mathrm{ml}$ venous blood sample was anticoagulated using EDTA-K2. Then, the blood was transferred into the centrifuge tube. The $3 \times$ Red 


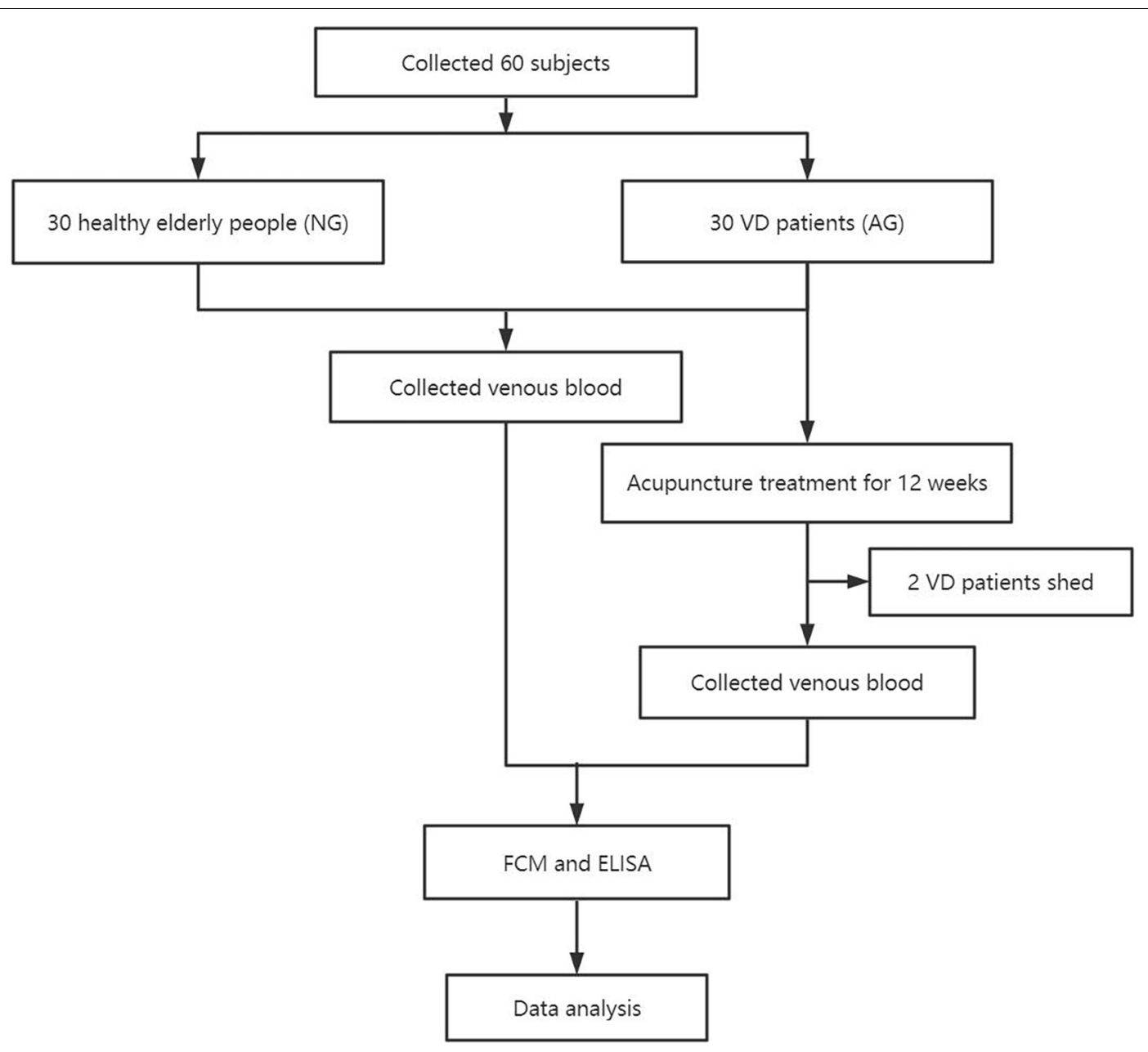

FIGURE 1| Research flowchart.

Blood Cell Lysis Buffer (Beijing Solarbio Science and Technology Co., Ltd., Beijing, China) was added into the tube and mixed thoroughly. Then, it was incubated at $4^{\circ} \mathrm{C}$ for $15 \mathrm{~min}$ in the dark (reverse mixing two times during the period). And, it was centrifuged at $450 \times g$ for $10 \mathrm{~min}$ to precipitate leukocytes. Later, the supernate was abandoned and the $2 \times$ Red Blood Cell Lysis Buffer was added into the leukocyte precipitation. It was mixed thoroughly and incubated at $4^{\circ} \mathrm{C}$ for $10 \mathrm{~min}$ in the dark (reverse mixing once during the period). Then, it was centrifuged at $450 \times g$ for $10 \mathrm{~min}$ and the supernate was abandoned. Finally, PBS was added into the tube to resuspend the cells which were then observed under the microscope. The cell concentration was adjusted to $10^{6}-10^{7}$ cells $/ \mathrm{ml}$ for cell staining. Then, according to the kit instructions, the cells were stained with different biomarker antibodies (Becton, Dickinson and Company, United States; eBioscience, United States). FACS Calibur (BD Biosciences, United States) was used to obtain data and Flow Jo 7.6.1 software (BD Biosciences, United States) was used for analysis.

This study used negative control for flow cytometry (FCM). The FCM gating strategy for TBNK is as follows: this study used four color schemes to analyze the lymphocyte subsets. When the cells were detected, the sample was divided into A tube and B tube. The A tube detected the PerCP-CD45, FITC-CD3, APCCD4, and PE-CD8, and the $\mathrm{B}$ tube detected the PerCP-CD45, FITC-CD3, APC-CD19, and PE-CD16 ${ }^{+} 56$. The specific gating strategy is shown in Figure 2. First, the lymphocyte gate was set up and named as R1 gate. Then, the lymphocytes (CD45 ${ }^{+}$cells) were confirmed by CD45/SSC, as shown in Figure 2A. Second, the cells were analyzed in the $\mathrm{R} 1$ gate. The T lymphocytes $\left(\mathrm{CD} 3^{+}\right.$ cells) were confirmed by CD3/SSC, as shown in Figure $\mathbf{2 B}$. The proportion of $\mathrm{CD} 4^{+} \mathrm{T}$ cells $\left(\mathrm{CD} 3^{+} / \mathrm{CD} 4^{+}\right.$cells) were determined by $\mathrm{CD} 3 / \mathrm{CD} 4$, as shown in Figure $2 \mathrm{C}$. The proportion of $\mathrm{CD}^{+}$ $\mathrm{T}$ cells $\left(\mathrm{CD}^{+} / \mathrm{CD}^{+}\right.$cells) were determined by $\mathrm{CD} 3 / \mathrm{CD} 8$, as shown in Figure 2D. Regarding the $\mathrm{B}$ tube, the first and second steps for the gating strategy were the same as for the A tube. Then, the B cells $\left(\mathrm{CD}^{-} / \mathrm{CD} 19^{+}\right.$cells) were confirmed by CD3/CD19, as shown in Figure 2E. The NK cells $\left(\mathrm{CD} 3^{-} / \mathrm{CD} 16^{+} 56^{+}\right)$were determined by $\mathrm{CD} 3 / \mathrm{CD}_{16}{ }^{+} 56$, as shown in Figure $2 \mathrm{~F}$. The flow cytometry gating strategy for Treg is shown in Figure 3. First, the lymphocytes were confirmed by FSC/SSC and named R1 gate, as shown in Figure 3A. Second, the cells were analyzed in the R1 gate. The $\mathrm{CD}^{+}$cells were determined by CD4/SSC and named as R2 gate, as shown in Figure 3B. Finally, the cells were analyzed in 


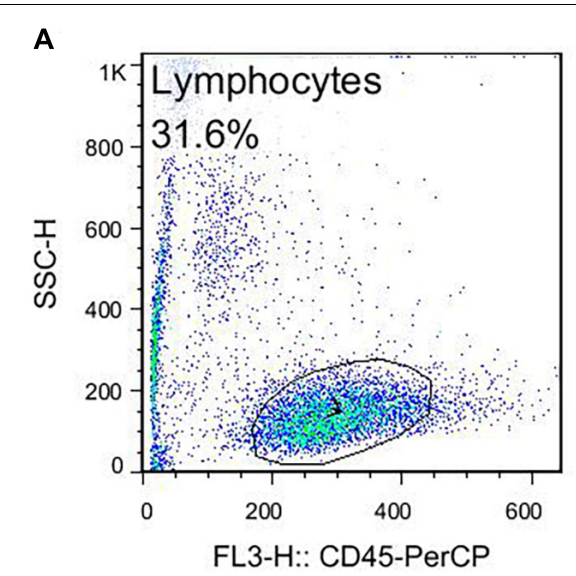

D

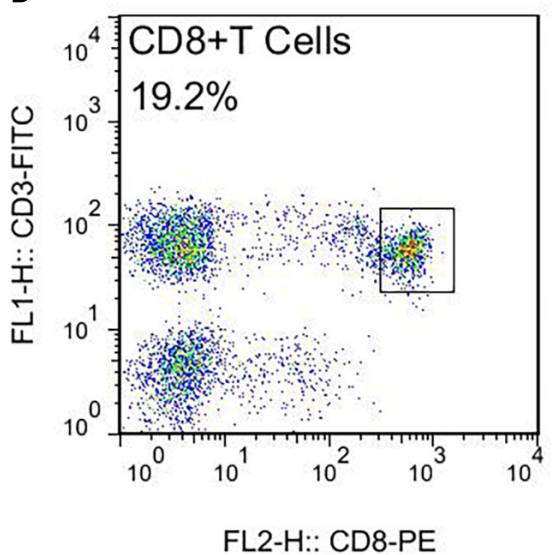

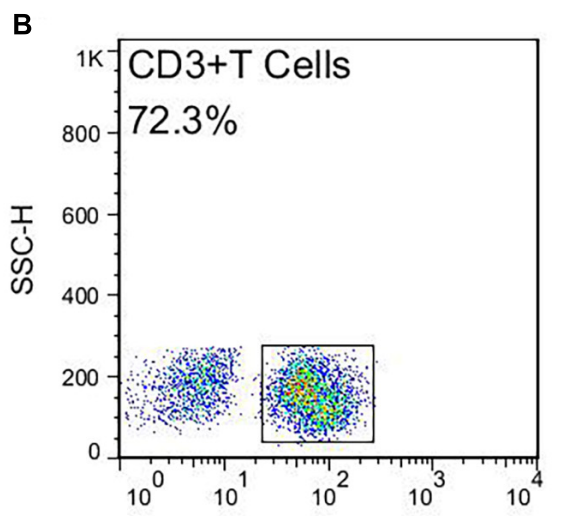

FL1-H:: CD3-FITC

E

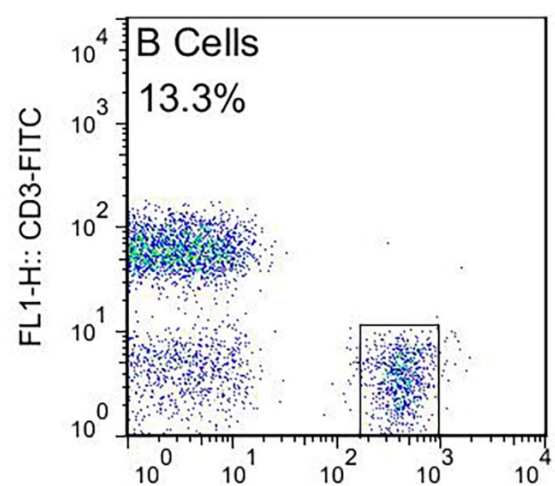

FL4-H:: CD19-APC

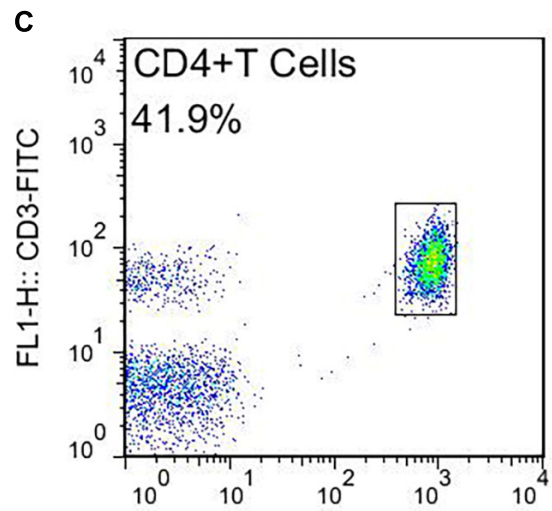

FL4-H:: CD4-APC

$\mathbf{F}$

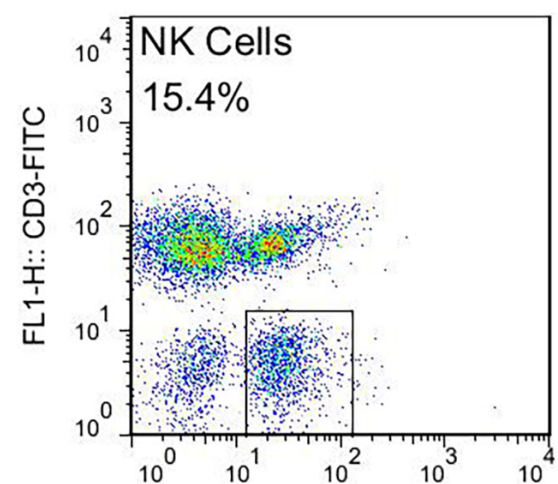

FL2-H:: CD16+56-PE

FIGURE 2 | The flow cytometry gating strategy for TBNK. (A) Lymphocytes. (B) T lymphocytes (CD3 ${ }^{+} \mathrm{T}$ cells). (C) The proportion of CD4 ${ }^{+} \mathrm{T}$ cells $\left(\mathrm{CD} 3^{+} \mathrm{CD} 4^{+}\right.$ T cells). (D) The proportion of CD8 ${ }^{+}$T cells (CD3 ${ }^{+}$CD8 ${ }^{+}$T cells). (E) The B cells (CD3-CD19+ B cells). (F) The NK cells (CD3-CD16 ${ }^{+}$CD56 ${ }^{+}$NK cells).

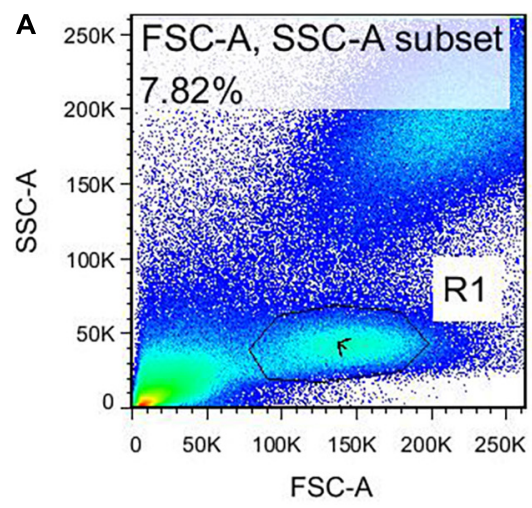

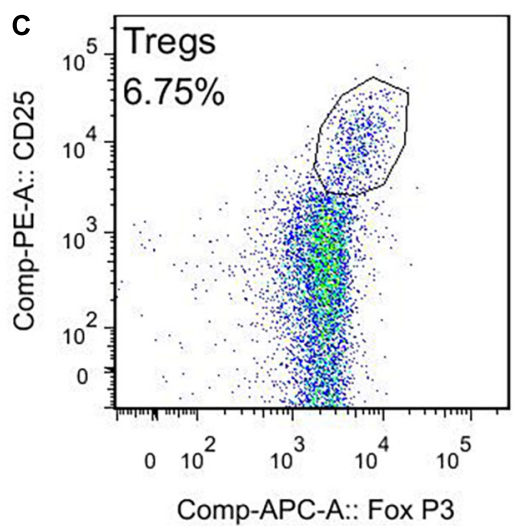

FIGURE 3 | The flow cytometry gating strategy for Treg. (A) Lymphocytes. (B) The CD4 ${ }^{+}$cells. (C) The Treg cells $\left(C D 4^{+}\right.$CD25 $5^{+}$Foxp3 ${ }^{+}$cells).

the R2 gate. The Treg cells (CD25+/Foxp $3^{+}$cells) were confirmed by Foxp3/CD25, as shown in Figure 3C.

Another $2 \mathrm{ml}$ venous blood was centrifuged at $4^{\circ} \mathrm{C} 1,500 \times g$ for $10 \mathrm{~min}$ to separate serum, and then the different cytokine levels in serum were detected using ELISA kits (IBL International $\mathrm{GmbH}$, Hamburg, Germany). The limits of detection were as follows: Human IL-1 0.3 pg/ml, Human IL-2 9.1 pg/ml, Human IL-4 1.3 pg/ml, Human IL-10 1.0 pg/ml, Human TNF- $\alpha 5.0$ $\mathrm{pg} / \mathrm{ml}$, and Human IFN- $\gamma 0.99 \mathrm{pg} / \mathrm{ml}$. Also, each sample was repeated three times in the test. In the $A G$, the blood of patients with VD was treated in the same way for testing before and after treatment. 


\section{Statistical Analysis}

All statistical analysis was performed using SPSS 21.0 (IBM Corporation, United States). Measurement data with normal distribution were expressed as mean \pm standard deviation $(\bar{x} \pm \mathrm{s})$, and measurement data with non-normal distribution were expressed as the median and interquartile range $(M$, Q). Measurement data for normal distribution between the two groups were tested using two independent samples, and measurement data for non-normal distribution were tested using a non-parametric test. The comparison of the conditions of patients before and after treatment was tested using paired sample $t$-test. Chi-square test was used for the comparison of count data, and a non-parametric test was used for the comparison of rank data. The significant difference level was $\alpha=0.05$. $P<0.05$ was considered statistically significant.

\section{RESULTS}

\section{Comparison of the General Conditions Before Treatment}

A total of 60 subjects were involved in this study, while 58 subjects completed the entire trial. Among them, the normal group included 30 healthy people, and the AG included 28 patients with VD. The baseline comparison of subjects of the two groups is shown in Table 1. It showed that among the different groups, there were no significant differences in gender, age, education level, etc. Also, they were comparable.

\section{Percentage of the Lymphocyte Subsets in Peripheral Blood}

Before treatment, the ratio of $\mathrm{CD}^{+}, \mathrm{CD}^{+}{ }^{+} \mathrm{T}$ cells, Tregs, B cells to total lymphocytes and $\mathrm{CD} 4^{+} / \mathrm{CD}^{+}$ratio of patients with $\mathrm{VD}$ were significantly lower compared with the normal group (all $P<0.05)$.

After the acupuncture treatment, the proportion of $\mathrm{CD}^{+}$, $\mathrm{CD}^{+} \mathrm{T}$ cells, and Tregs in the peripheral blood of patients with $\mathrm{VD}$ in the AG was significantly increased compared with that before treatment (all $P<0.05$ ). There was no significant change in the proportion of $\mathrm{CD}^{+} \mathrm{T}$ cells, $\mathrm{CD} 4^{+} / \mathrm{CD} 8^{+}, \mathrm{B}$ cells, and $\mathrm{NK}$ cells after treatment in the AG (all $P>0.05$ ).

In contrast, after the acupuncture treatment, the proportion of Tregs in patients with VD was still low compared with the normal group $(P<0.05)$. And after the treatment, the proportion of $\mathrm{CD}^{+}, \mathrm{CD}^{+} \mathrm{T}$ cells, B cells, and the ratio of $\mathrm{CD} 4^{+} / \mathrm{CD}^{+}$ were slightly lower compared with the normal group, but there was no statistical difference between the two groups (all $P>0.05)$. The proportion of $\mathrm{CD}^{+} \mathrm{T}$ cells was slightly higher, but there was no statistical difference between the two groups $(P>0.05)$. There was no significant difference in the proportion of NK cells compared with the normal group after treatment $(P>0.05)$ (Figures 4, 5).

\section{Cytokine Levels in Peripheral Blood}

Before treatment, the levels of IL-10 and IFN- $\gamma$ in the peripheral blood of patients with VD were significantly reduced compared with the normal group $(P<0.05)$. The content of TNF- $\alpha$ was increased significantly $(P<0.05)$. There were no significant changes in IL-1 $\beta$, IL-2, and IL-4 between the two groups $(P>0.05)$.

After acupuncture treatment, the levels of IL-4 and TNF- $\alpha$ in the peripheral blood of patients with VD were significantly reduced in the $\mathrm{AG}$ compared with that before treatment $(P<0.05)$. The IFN- $\gamma$ level was significantly increased in the AG $(P<0.05)$. The levels of IL-1 $\beta$ and IL-2 of patients with VD showed a downward trend, but there was no statistical significance after treatment $(P>0.05)$. There was no significant change in IL-10 after treatment $(P>0.05)$.

Compared with the healthy elderly people, the level of IL10 was still lower after acupuncture treatment $(P<0.05)$. The contents of IL- $1 \beta$, IL- 2 , IL- 4 , TNF- $\alpha$, and IFN- $\gamma$ were slightly lower, and there was no statistical difference between the two groups $(P>0.05)$ (Figure 6).

\section{DISCUSSION}

Our previous studies had shown that the "sanjiao" acupuncture method could significantly improve the cognitive impairment and behavioral capacity of patients with VD. Also, there were no adverse effects caused by the acupuncture treatment ( $\mathrm{Yu}$ et al., 2006). Moreover, during the course of this study, the cognitive function of patients with VD was improved after the acupuncture treatment, and they had no significantly related adverse reactions except for the minor bleeding caused by needle acupuncture, which suggested that acupuncture treatment for VD was safe and effective. Hence, this study further investigated the regulatory effect of acupuncture treatment on the peripheral immune function of patients with VD and confirmed that the "sanjiao" acupuncture method could increase the proportion of $\mathrm{CD}^{+}, \mathrm{CD}^{+} \mathrm{T}$ cells, and Tregs in the peripheral blood of patients with VD. Also, it reduced the levels of pro-inflammatory factor TNF- $\alpha$, which achieved the anti-inflammatory effects and immunostimulation. This suggested that acupuncture could improve the peripheral immune dysfunction of patients with VD by regulating the distribution of lymphocyte subsets and the level of inflammatory cytokines.

Vascular dementia is the second most common type of dementia after Alzheimer's disease, and it is caused by a decrease in cerebral blood flow and damage to the neurovascular unit (Sinha et al., 2020). There is no doubt that neuroinflammation plays a critical role in the occurrence and progress of VD (Iadecola, 2010). However, in recent years, the promotion of peripheral inflammation on the neuroinflammation, and the important role of the peripheral immune cells, especially $\mathrm{T}$ cells, in maintaining normal cognitive function, have been paid more attention. Evidence indicates that there are peripheral inflammation and abnormal immune function in $\mathrm{VD}$, which are closely associated with cognitive disorder (Schmitz et al., 2015; Busse et al., 2017; Guo et al., 2019). Research shows that lipopolysaccharide (LPS) injection can activate microglial cells and result in an increased production of IL- $1 \beta$ and TNF- $\alpha$ in the rat hippocampus, which finally induces learning and memory 
TABLE 1 | Comparison of general conditions of the two groups.

\begin{tabular}{|c|c|c|c|c|c|}
\hline & Normal group $(n=30)$ & Acupuncture group $(n=28)$ & Aggregate & Statistic & $P$-value \\
\hline \multicolumn{6}{|l|}{ Gender: Number (\%) } \\
\hline Male & $13(43.33)$ & $16(57.14)$ & $29(50)$ & $X^{2}=1.105$ & 0.293 \\
\hline Female & $17(56.67)$ & $12(42.86)$ & $29(50)$ & & \\
\hline $\operatorname{Age}(\bar{x} \pm s)$ & $68.53 \pm 6.68$ & $68.14 \pm 6.90$ & & $t=-0.219$ & 0.828 \\
\hline \multicolumn{6}{|l|}{ Education level: Number (\%) } \\
\hline Illiterate (uneducated) & $1(3.33)$ & $2(7.14)$ & $3(5.17)$ & $Z=-0.314$ & 0.754 \\
\hline Primary school (education period $\leq 6$ years) & $6(20)$ & $4(14.29)$ & $10(17.24)$ & & \\
\hline Secondary school (6 years < education period $\leq 12$ years) & $20(66.67)$ & 18 (64.29) & 38 (65.52) & & \\
\hline University (education period > 12 years) & $3(10)$ & $4(14.29)$ & $7(12.07)$ & & \\
\hline
\end{tabular}

deficits (Tanaka et al., 2006). The hippocampus nerve of the immunodeficient mice is significantly impaired, and it cannot be induced by a complex environment. The structure and function of the hippocampal nerve can recover after $\mathrm{T}$ cells eliminated the specific central nervous system antigen, which indicates that $\mathrm{T}$ cells are the foundation of hippocampal plasticity and cell renewal. Research shows that the $\mathrm{CD}^{+} \mathrm{T}$ cells are the most important immune cells for learning. The learning ability of rats is decreased after using anti-CD4, but not after using anti-CD8 (Prinz and Priller, 2017). The $\mathrm{T}$ cells, especially CD $4^{+} \mathrm{T}$ cells, may be the most important lymphocyte subsets in maintaining normal cognitive function.

\section{Distribution of Lymphocyte Subsets in the Peripheral Blood of Patients With Vascular Dementia}

A study shows that VD has a pronounced impact on the peripheral immunity. The $\mathrm{CD}^{+}, \mathrm{CD}^{+}$, and $\mathrm{CD}^{+} \mathrm{T}$ cells, $\mathrm{B}$ cells, and $\mathrm{NK}$ cells are severely decreased in patients with VD (Busse et al., 2017). Our study found that before treatment, the proportion of $\mathrm{CD}^{+}$and $\mathrm{CD}^{+} \mathrm{T}$ cells, and the ratio of $\mathrm{CD}^{+} / \mathrm{CD}^{+}$in patients with $\mathrm{VD}$ were significantly lower than those in normal elderly people. This result was similar to the previous studies, which suggested that the peripheral immune function of patients with VD had decreased. And after acupuncture treatment, the proportion of $\mathrm{CD}^{+}$and $\mathrm{CD}^{+} \mathrm{T}$ cells in the peripheral blood was increased, indicating that acupuncture could enhance cellular immune function.

Regulatory $\mathrm{T}$ cell (Treg) is a special $\mathrm{CD}^{+}{ }^{+} \mathrm{T}$ cell subset that can maintain immune homeostasis, protect autoimmunity, and prevent excessive inflammation (Pacella and Piconese, 2019). On the one hand, abnormal Tregs can induce more production of the pro-inflammatory factors (IL-6, IL-17, and IFN- $\gamma$ ), then aggravate the neuroinflammation and cognitive disorder (Van Mierlo et al., 2008). On the other hand, Tregs contribute to the recovery and regeneration of the central nervous system (Zhang et al., 2017; Jiatao et al., 2018). Research indicates that the increased expression of IL- 6 and the decline of Tregs may be related to the cognitive disorder of patients with vascular cognitive impairment (VCI) (Guoping et al., 2015). Our study also finds that the percentage of Tregs in the peripheral blood of patients with VD was lower than that of normal elderly people, resulting in the immune inflammation response, thereby affecting the normal immune function of patients with VD. After the acupuncture treatment, the proportion of Tregs increased, indicating that acupuncture treatment could reduce the immuneinflammatory response, reduce the inflammatory damage of tissues and organs, and improve the immune function of patients with VD.

Regarding the B cells and NK cells, the results of our study showed that the proportion of B cells in the peripheral blood of patients with VD was significantly lower than that of normal elderly people, while NK cells were not significantly changed. B cells are mainly involved in humoral immunity, so a decrease in the number of $\mathrm{B}$ cells indicates that there is a decline in humoral immunity of patients with VD. However, there was no significant change in the proportion of B cells and NK cells after acupuncture treatment in our study, which may be because the sample size of this study is small, and this part needs further research.

\section{Cytokines Expression in the Peripheral Blood of Patients With Vascular Dementia}

Interleukin is one of the inflammatory cytokines related to VD, which is involved in the development and pathophysiology of VD (Chang et al., 2011). The researchers observed a significantly increased level of IL-1 $\beta$, TNF- $\alpha$, IFN- $\gamma$, IL-4, and IL-5 (Schmitz et al., 2015). However, the results of our study showed that the levels of IL-1 $\beta$, IL-2, and IL-4 in the peripheral blood of patients with VD were not significantly different from those of normal elderly people, which may be due to the small sample size of this study and the degree of dementia in this trial was mild compared with other studies. Other related clinical studies are rarely reported, indicating that this part needs further exploration and research.

A study finds that the IL-10 level in the cerebrospinal fluid (CSF) is decreased in VD (Kaiser et al., 2014). Our study also found that the level of IL-10 in the peripheral blood of patients with VD was significantly reduced compared with that of the normal people before treatment. It could be speculated that the decreased expression of IL-10 in the peripheral blood of patients with VD enhanced the inflammatory response, which played a crucial role in the progress of VD. 


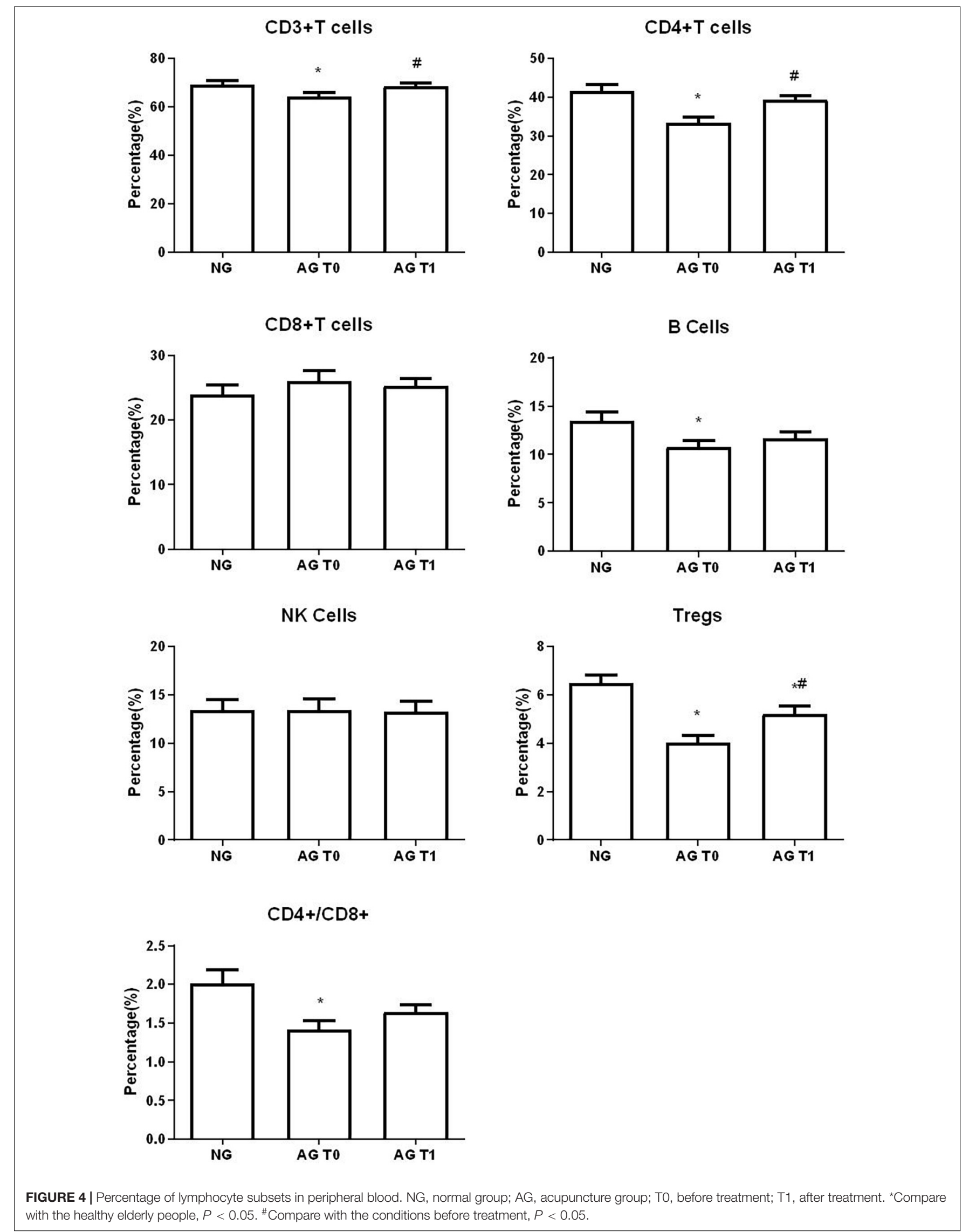




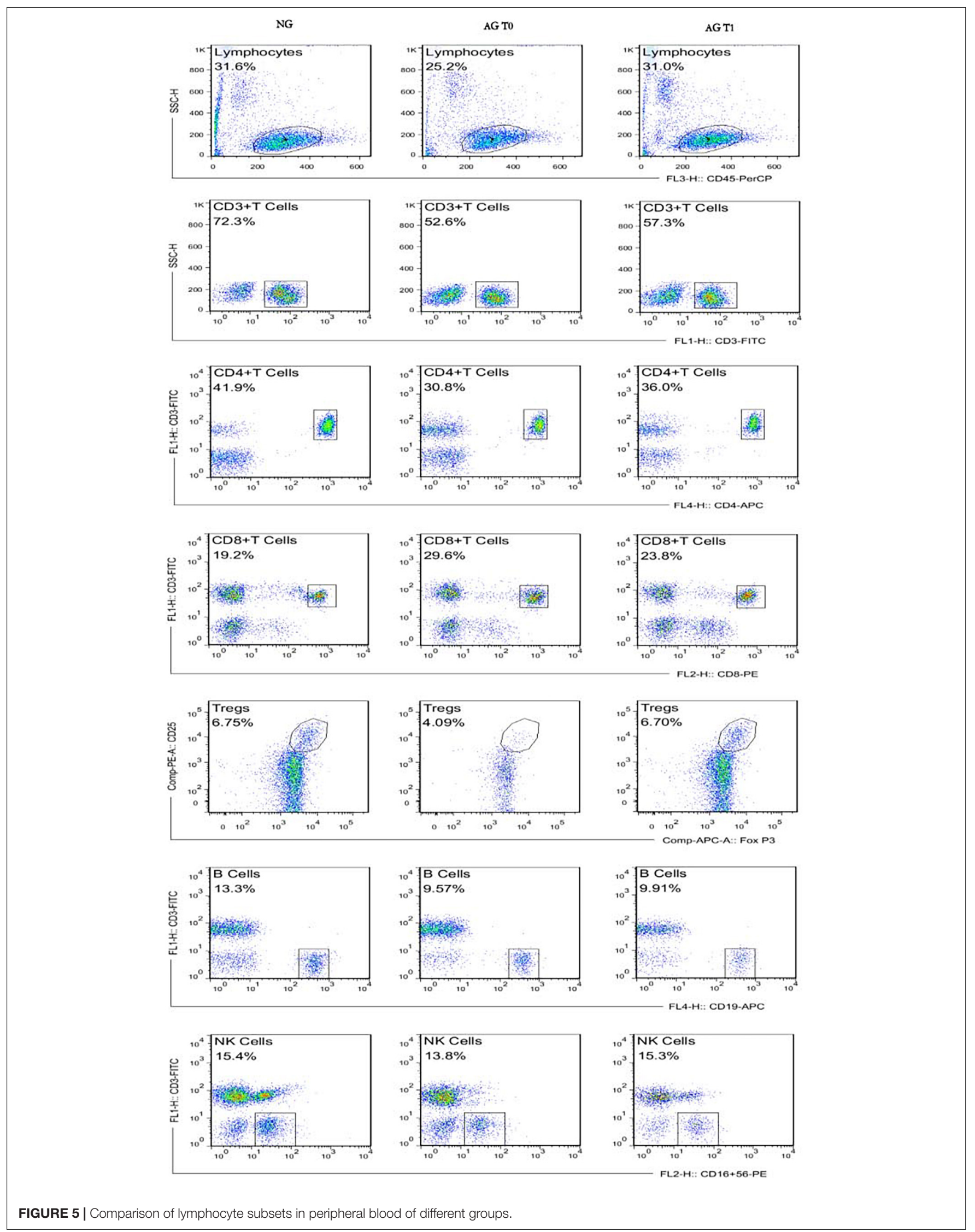




\section{IL-1beta}

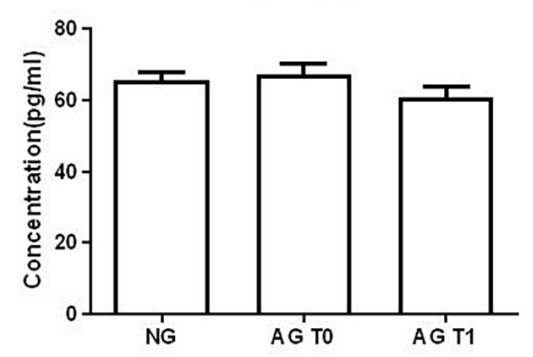

IL-10

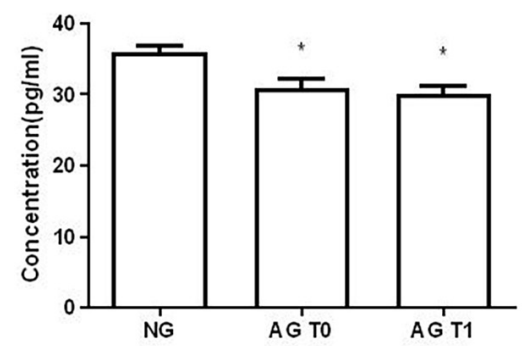

IL-2

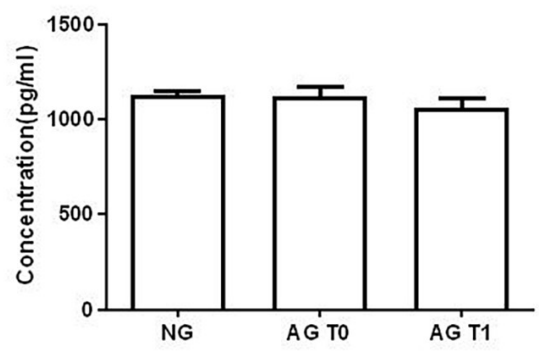

TNF-alpha

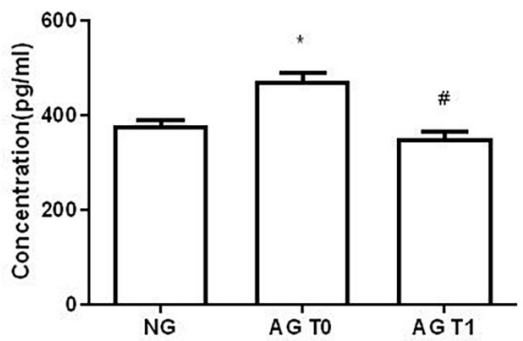

IL-4

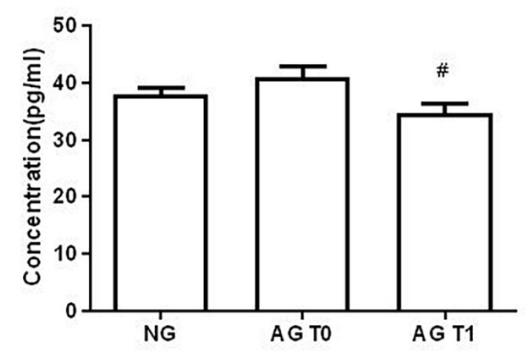

IFN-gamma

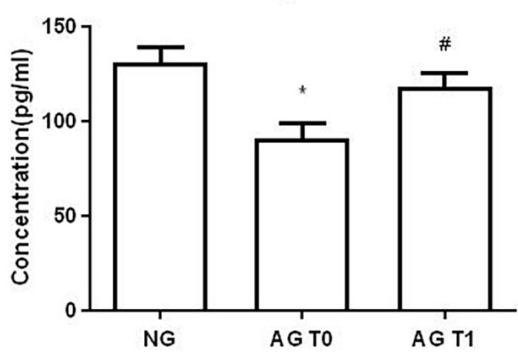

FIGURE 6 | Cytokine concentration in peripheral blood. *Compare with the healthy elderly people, $P<0.05$. "Compare with the conditions before treatment, $P<0.05$.

A study reports that the pro-inflammatory cytokines TNF- $\alpha$, IL-6, IL-1 $\beta$, IL-2, and IL-18 in the peripheral blood samples of patients with $\mathrm{AD}$ are increased significantly (Lai et al., 2017). Elevated serum TNF- $\alpha$ is confirmed in both patients with VD and VD rat models, and it is closely related to the degree of dementia and prognosis of patients (Sheng et al., 2012; Liu et al., 2014). Studies show that TNF- $\alpha$ is closely related to cognitive function, and the elevation of TNF- $\alpha$ can cause acute cognitive disorder (Hennessy et al., 2017; Magalhães et al., 2018). Otherwise, research shows that the increase of TNF- $\alpha$ level in peripheral blood is related to the decrease of hippocampal volume (Sudheimer et al., 2014), which indicates that the TNF- $\alpha$ level plays a key role in the neurodegeneration in the hippocampus. Our study also found that the TNF- $\alpha$ level in the peripheral blood of patients with VD was increased, which was consistent with the results obtained in previous studies. After the acupuncture treatment, the TNF- $\alpha$ level was decreased, indicating that acupuncture could improve the peripheral inflammation.

IFN- $\gamma$ is a kind of cytokine, which is released by Th1 cells, cytotoxic T cells, and NK cells (Mosser and Edwards, 2008; Colton, 2009). Some studies discover that the increased expression levels of IL-6 and IFN- $\gamma$ are bound up with deteriorating cognitive deficits in patients with VCI (Guoping et al., 2015). But some other studies have shown that patients with stroke have a rapid decrease in $\mathrm{T}$ lymphocytes and a persistent decrease in INF- $\gamma$ level (Klehmet et al., 2009). Otherwise, under the pathological conditions, IFN- $\gamma$ plays an important role in the activation of microglial cells. It can cause the overreaction of microglial cells, induce the proliferation and activation of microglial cells, then cause the release of pro-inflammatory factors and cytotoxic substances, and result in neuronal death in the end (Colonna and Butovsky, 2017; Prinz and Priller, 2017). Some studies find that the effect of IFN- $\gamma$ on ischemic stroke may be a double-edged sword. On the one hand, it can cause inflammation after cerebral ischemia and aggravate the damage. On the other hand, it has the neuroprotective and neuroregulation effect to some extent. And it can influence the activation of endogenous nerve cells (Wong et al., 2004; Cossetti et al., 2014; LlorensBobadilla et al., 2015; Fantetti et al., 2016). The results of our study suggested that compared with the normal subjects, the IFN- $\gamma$ level in the peripheral blood of patients with VD was significantly reduced before treatment. After the acupuncture treatment, the IFN- $\gamma$ level in the peripheral blood of patients with VD was increased, but it was still lower than that of normal people. The clinical research results about the IFN $-\gamma$ are contradictory and the effect of IFN- $\gamma$ is unclear, which needs to be further explored.

\section{"Sanjiao" Acupuncture Method for Vascular Dementia}

As a part of traditional Chinese medicine, acupuncture has unique advantages in treating VD (Shi et al., 2012). A study shows that acupuncture can improve the behavior and psychological symptoms of dementia (Harris et al., 2019). Acupuncture plays an integral role in antioxidation, anti-apoptosis effect, improving cerebral blood flow, and mitochondrial dysfunction in VD (Wang et al., 2009; Zhang et al., 2014; Li et al., 2015).

Professor Han Jingxian summed up his many years of clinical experience and created the "sanjiao" acupuncture method. The prescription included “Danzhong” (CV17), "Zhongwan” (CV12), 
"Qiha” (CV6), bilateral "Xuehai” (SP10), "Zusanli” (ST36), and "waiguan" (SJ5) six acupoints. It could treat a variety of senile diseases and achieve good clinical results. Previous studies have shown that the "sanjiao" acupuncture method can significantly improve cognitive impairment and behavioral capacity in patients with VD (Yu et al., 2006).

On the one hand, this study further confirmed that the "sanjiao" acupuncture method could increase the proportion of $\mathrm{CD}^{+}, \mathrm{CD}^{+} \mathrm{T}$ cells, and Tregs in the peripheral blood of patients with VD. On the other hand, it reduced the levels of pro-inflammatory factor $\mathrm{TNF}-\alpha$, which achieved the anti-inflammatory effects and immunostimulation. Antiinflammatory treatment could reduce the inflammatory damage of tissues and organs, and it could also improve immune function. Immunostimulation reduced the inflammatory response by improving immune function. Therefore, acupuncture achieved the effect of improving peripheral immune function in patients with VD.

\section{CONCLUSION}

The acupuncture method can increase the proportion of $\mathrm{CD}^{+}$, $\mathrm{CD} 4{ }^{+} \mathrm{T}$ cells, and Tregs in the peripheral blood of patients with VD. Also, it reduces the levels of pro-inflammatory factor TNF- $\alpha$ which achieves the anti-inflammatory effects and immunostimulation. It is suggests that acupuncture can improve the peripheral immune dysfunction of patients with VD by regulating the distribution of lymphocyte subsets and the levels of inflammatory cytokines.

\section{REFERENCES}

An, C., Shi, Y., Li, P., Hu, X., Gan, Y., Stetler, R. A., et al. (2014). Molecular dialogs between the ischemic brain and the peripheral immune system: dualistic roles in injury and repair. Prog. Neurobiol. 115, 6-24. doi: 10.1016/j.pneurobio.2013. 12.002

Balzano, T., Dadsetan, S., Forteza, J., Cabrera-Pastor, A., Taoro-González, L., Malaguarnera, M., et al. (2020). Chronic hyperammonemia induces peripheral inflammation that leads to cognitive impairment in rats: reversed by anti-TNF $\alpha$ treatment. J. Hepatol. 73, 582-592. doi: 10.1016/j.jhep.2019.01.008

Balzano, T., Dadsetan, S., Forteza, J., Cabrera-Pastor, A., Taoro-González, L., Malaguarnera, M., et al. (2019). Chronic hyperammonemia induces peripheral inflammation that leads to cognitive impairment in rats: reversal by anti-tnfa treatment. J. Hepatol. 73, 582-592.

Busse, M., Michler, E., Von Hoff, F., Dobrowolny, H., Hartig, R., Frodl, T., et al. (2017). Alterations in the peripheral immune system in dementia. J. Alzheimers Dis. 58, 1303-1313. doi: 10.3233/JAD-161304

Chang, X., Hou, X., Pan, J., Fang, K., Wang, L., and Han, J. (2011). Investigating the pathogenic role of PADI4 in oesophageal cancer. Int. J. Biol. Sci. 7, 769-781.

Chen, L., Xu, A., Yin, N., Zhao, M., Wang, Z., Chen, T., et al. (2017). Enhancement of immune cytokines and splenic CD4+ T cells by electroacupuncture at ST36 acupoint of SD rats. PLoS One 12:e0175568. doi: 10.1371/journal.pone.0175568

Chen, Z., Chunhui, B., Jing, L., Zhu, Y., Wang, S., Yang, L., et al. (2015). Moxibustion and acupuncture ameliorate Crohn's disease by regulating the balance between Th17 and treg cells in the intestinal mucosa. Evid. Based Complement. Alternat. Med. 2015:938054. doi: 10.1155/2015/938054

Colonna, M., and Butovsky, O. (2017). Microglia function in the central nervous system during health and neurodegeneration. Annu. Rev. Immunol. 35, 441468. doi: 10.1146/annurev-immunol-051116-052358

\section{DATA AVAILABILITY STATEMENT}

The raw data supporting the conclusions of this article will be made available by the authors, without undue reservation.

\section{ETHICS STATEMENT}

The studies involving human participants were reviewed and approved by the Research Ethical Committee of First Teaching Hospital of Tianjin University of Traditional Chinese Medicine. The patients/participants provided their written informed consent to participate in this study.

\section{AUTHOR CONTRIBUTIONS}

$\mathrm{XZ}, \mathrm{YW}$, and SC participated in the design of the study. YW and SC carried out the acupuncture manipulation and analyzed the data. ZM and RW collected the sample. YW, SC, PP, ZL, and $\mathrm{ZZ}$ evaluated the sample of the subjects. $\mathrm{HZ}$ and $\mathrm{YW}$ wrote the manuscript. All authors contributed to the article and approved the submitted version.

\section{FUNDING}

This research was supported by the National Natural Science Foundation of China $(81473766,82174492)$ and the Science and technology support program of Tianjin City (20YFZCSY00810).

Colton, C. A. (2009). Heterogeneity of microglial activation in the innate immune response in the brain. J. Neuroimmune Pharmacol. 4, 399-418. doi: 10.1007/ s11481-009-9164-4

Cossetti, C., Iraci, N., Mercer, T. R., Leonardi, T., Alpi, E., Drago, D., et al. (2014). Extracellular vesicles from neural stem cells transfer IFN-gamma via Ifngrl to activate stat1 signaling in target cells. Mol. Cell 56, 193-204. doi: 10.1016/j. molcel.2014.08.020

Dadsetan, S., Balzano, T., Forteza, J., Cabrera-Pastor, A., Taoro-Gonzalez, L., Hernandez-Rabaza, V., et al. (2016). Reducing peripheral inflammation with infliximab reduces neuroinflammation and improves cognition in rats with hepatic encephalopathy. Front. Mol. Neurosci. 9:106. doi: 10.3389/fnmol.2016. 00106

De Miranda, A. S., Zhang, C. J., Katsumoto, A., and Teixeira, A. L. (2017). Hippocampal adult neurogenesis: does the immune system matter? J. Neurol. Sci. 372, 482-495. doi: 10.1016/j.jns.2016.10.052

Dichgans, M., and Leys, D. (2017). Vascular cognitive impairment. Circ. Res. 120, 573-591. doi: 10.1161/CIRCRESAHA.116.308426

Fantetti, K. N., Gray, E. L., Ganesan, P., Kulkarni, A., and O’Donnell, L. A. (2016). Interferon gamma protects neonatal neural stem/progenitor cells during measles virus infection of the brain. J. Neuroinflammation 13:107. doi: 10.1186/ s12974-016-0571-1

Guo, Z., Peng, X., Li, H. Y., Wang, Y., Qian, Y., Wang, Z., et al. (2019). Evaluation of peripheral immune dysregulation in Alzheimer's disease and vascular dementia. J. Alzhmers Dis. JAD 71, 1-12. doi: 10.3233/JAD-19 0666

Guoping, P., Wei, W., Xiaoyan, L., Fangping, H., Zhongqin, C., and Benyan, L. (2015). Characteristics of the peripheral T cell immune response of patients at different stages of vascular cognitive impairment. Immunol. Lett. 168, 120-125. doi: 10.1016/j.imlet.2015.09.015 
Harris, M. L., Titler, M. G., and Struble, L. M. (2019). Acupuncture and acupressure for dementia behavioral and psychological symptoms: a scoping review. West. J. Nurs. Res. 42, 867-880. doi: 10.1177/0193945919890552

Hennessy, E., Gormley, S., Lopez-Rodriguez, A. B., Murray, C., Murray, C., and Cunningham, C. (2017). Systemic TNF $\alpha$ produces acute cognitive dysfunction and exaggerated sickness behavior when superimposed upon progressive neurodegeneration. Brain Behav. Immun. 59, 233-244. doi: 10.1016/j.bbi.2016. 09.011

Iadecola, C. (2010). The overlap between neurodegenerative and vascular factors in the pathogenesis of dementia. Acta Neuropathol. 120, 287-296. doi: 10.1007/ s00401-010-0718-6

Jiatao, L., Jean, T., Martino, M. M., and Lui, K. O. (2018). Regulatory T-Cells: potential regulator of tissue repair and regeneration. Front. Immunol. 9:585. doi: 10.3389/fimmu.2018.00585

Kaiser, D., Weise, G., Moller, K., Scheibe, J., Posel, C., Baasch, S., et al. (2014). Spontaneous white matter damage, cognitive decline and neuroinflammation in middle-aged hypertensive rats: an animal model of early-stage cerebral small vessel disease. Acta Neuropathol. Commun. 2:169. doi: 10.1186/s40478-0140169-8

Klehmet, J., Harms, H., Richter, M., Prass, K., Volk, H. D., Dirnagl, U., et al. (2009). Stroke-induced immunodepression and post-stroke infections: lessons from the preventive antibacterial therapy in stroke trial. Neuroscience 158, 1184-1193. doi: 10.1016/j.neuroscience.2008.07.044

Lai, K. S. P., Liu, C. S., Rau, A., Lanctot, K. L., Kohler, C. A., Pakosh, M., et al. (2017). Peripheral inflammatory markers in Alzheimer's disease: a systematic review and meta-analysis of 175 studies. J. Neurol. Neurosurg. Psychiatry 88, 876-882. doi: 10.1136/jnnp-2017-316201

Li, H., Liu, Y., Lin, L. T., Wang, X. R., Du, S. Q., Yan, C. Q., et al. (2015). Acupuncture reversed hippocampal mitochondrial dysfunction in vascular dementia rats. Neurochem. Int. 92, 35-42. doi: 10.1016/j.neuint.2015.12.001

Liu, J., Li, J., Yang, Y., Wang, X., Zhang, Z., and Zhang, L. (2014). Neuronal apoptosis in cerebral ischemia/reperfusion area following electrical stimulation of fastigial nucleus. Neural Regen. Res. 9, 727-734. doi: 10.4103/1673-5374. 131577

Llorens-Bobadilla, E., Zhao, S., Baser, A., Saiz-Castro, G., Zwadlo, K., and MartinVillalba, A. (2015). Single-Cell transcriptomics reveals a population of dormant neural stem cells that become activated upon brain injury. Cell Stem Cell 17, 329-340. doi: 10.1016/j.stem.2015.07.002

Magalhães, C. A., Ferreira, C. N., Loures, C. M. G., Fraga, V. G., Chaves, A. C., Oliveira, A. C. R., et al. (2018). Leptin, hsCRP, TNF $\alpha$ and IL-6 levels from normal aging to dementia: relationship with cognitive and functional status. J. Clin. Neurosci. 56, 150-155. doi: 10.1016/j.jocn.2018.08.027

Mosser, D. M., and Edwards, J. P. (2008). Exploring the full spectrum of macrophage activation. Nat. Rev. Immunol. 8, 958-969. doi: 10.1038/nri2448

O'Brien, J. T., and Thomas, A. (2015). Vascular dementia. Lancet 386, 1698-1706. doi: 10.1016/S0140-6736(15)00463-8

Pacella, I., and Piconese, S. (2019). Immunometabolic checkpoints of treg dynamics: adaptation to microenvironmental opportunities and challenges. Front. Immunol. 10:1889. doi: 10.3389/fimmu.2019.01889

Prinz, M., and Priller, J. (2017). The role of peripheral immune cells in the CNS in steady state and disease. Nat. Neurosci. 20, 136-144. doi: 10.1038/nn.4475

Radjavi, A., Smirnov, I., and Kipnis, J. (2014). Brain antigen-reactive CD4+ T cells are sufficient to support learning behavior in mice with limited $\mathrm{T}$ cell repertoire. Brain Behav. Immun. 35, 58-63. doi: 10.1016/j.bbi.2013.08.013

Raz, L., Knoefel, J., and Bhaskar, K. (2015). The neuropathology and cerebrovascular mechanisms of dementia. J. Cereb. Blood Flow Metab. 36, 172-186. doi: 10.1038/jcbfm.2015.164

Schmitz, M., Hermann, P., Oikonomou, P., Stoeck, K., Ebert, E., Poliakova, T. G., et al. (2015). Cytokine profiles and the role of cellular prion protein in patients with vascular dementia and vascular encephalopathy. Neurobiol. Aging 36, 2597-2606. doi: 10.1016/j.neurobiolaging.2015.05.013

Sheng, R., Liu, X. Q., Zhang, L. S., Gao, B., Han, R., Wu, Y. Q., et al. (2012). Autophagy regulates endoplasmic reticulum stress in ischemic preconditioning. Autophagy 8, 310-325. doi: 10.4161/auto.18673
Shi, G. X., Liu, C. Z., Li, Q. Q., Zhu, H., and Wang, L. (2012). Influence of acupuncture on cognitive function and markers of oxidative DNA damage in patients with vascular dementia. J. Tradit. Chin. Med. 32, 199-202. doi: 10.1016/S0254-6272(13)60011-4

Si, Y. C., Song, Y. L., Niu, X., and Wan, F. (2016). The role of Chinese herbs and acupuncture on the inflammation reaction after cerebral Ischemia. Curr. Pharm. Design 22, 709-719. doi: 10.2174/138161282266615120400 1348

Sinha, K., Sun, C., Kamari, R., and Bettermann, K. (2020). Current status and future prospects of pathophysiology-based neuroprotective drugs for the treatment of vascular dementia. Drug Discov. Today 25, 793-798. doi: 10.1016/j.drudis.2020. 01.003

Sudheimer, K. D., O’Hara, R., Spiegel, D., Powers, B., Kraemer, H. C., Neri, E., et al. (2014). Cortisol, cytokines, and hippocampal volume interactions in the elderly. Front. Aging Neurosci. 6:153. doi: 10.3389/fnagi.2014.00153

Tanaka, S., Ide, M., Shibutani, T., Ohtaki, H., Numazawa, S., Shioda, S., et al. (2006). Lipopolysaccharide-induced microglial activation induces learning and memory deficits without neuronal cell death in rats. J. Neurosci. Res. 83, 557-566. doi: 10.1002/jnr.20752

Van Mierlo, G. J. D., Scherer, H. U., Hameetman, M., Morgan, M. E., Flierman, R., Huizinga, T. W. J., et al. (2008). Cutting edge: TNFR-Shedding by CD4+CD25+ regulatory $\mathrm{T}$ cells inhibits the induction of inflammatory mediators. J. Immunol. 180, 2747-2751. doi: 10.4049/jimmunol.180.5.2747

Venkat, P., Chopp, M., and Chen, J. (2015). Models and mechanisms of vascular dementia. Exp. Neurol. 272, 97-108. doi: 10.1016/j.expneurol.2015.05.006

Wang, T., Liu, C. Z., Yu, J. C., Jiang, W., and Han, J. (2009). Acupuncture protected cerebral multi-infarction rats from memory impairment by regulating the expression of apoptosis related genes Bcl-2 and Bax in hippocampus. Physiol. Behav. 96, 155-161. doi: 10.1016/j.physbeh.2008.09.024

Wong, G., Goldshmit, Y., and Turnley, A. M. (2004). Interferon-gamma but not TNF alpha promotes neuronal differentiation and neurite outgrowth of murine adult neural stem cells. Exp. Neurol. 187, 171-177. doi: 10.1016/j.expneurol. 2004.01.009

Yu, J., Zhang, X., Liu, C., Meng, Y., and Han, J. (2006). Effect of acupuncture treatment on vascular dementia. Neurol. Res. 28:97. doi: 10.1179/ 016164106 X91951

Zhang, C., Li, L., Feng, K., Fan, D., Xue, W., and Lu, J. (2017). 'Repair' Treg cells in tissue injury. Cell. Physiol. Biochem. 43, 2155-2169. doi: 10.1159/00048 4295

Zhang, X., Wu, B., Nie, K., Jia, Y., and Yu, J. (2014). Effects of acupuncture on declined cerebral blood flow, impaired mitochondrial respiratory function and oxidative stress in multi-infarct dementia rats. Neurochem. Int. 65, 23-29. doi: 10.1016/j.neuint.2013.12.004

Zhao, J., Bi, W., Xiao, S., Lan, X., Cheng, X., Zhang, J., et al. (2019). Neuroinflammation induced by lipopolysaccharide causes cognitive impairment in mice. Sci. Rep. 9:5790. doi: 10.1038/s41598-019-42286-8

Conflict of Interest: The authors declare that the research was conducted in the absence of any commercial or financial relationships that could be construed as a potential conflict of interest.

Publisher's Note: All claims expressed in this article are solely those of the authors and do not necessarily represent those of their affiliated organizations, or those of the publisher, the editors and the reviewers. Any product that may be evaluated in this article, or claim that may be made by its manufacturer, is not guaranteed or endorsed by the publisher.

Copyright (c) 2021 Zhi, Wang, Chang, Pan, Ling, Zhang, Ma, Wang and Zhang. This is an open-access article distributed under the terms of the Creative Commons Attribution License (CC BY). The use, distribution or reproduction in other forums is permitted, provided the original author(s) and the copyright owner(s) are credited and that the original publication in this journal is cited, in accordance with accepted academic practice. No use, distribution or reproduction is permitted which does not comply with these terms. 